Association for Information Systems AIS Electronic Library (AISeL)

2005

\title{
Information Search Patterns in E-Commerce Product Comparison Services
}

Fiona Fui-Hoon Nah

University of Nebraska - Lincoln, fnah@unlnotes.unl.edu

Hong-Hee Lee

University of Nebraska - Lincoln, honghlee@bigred.unl.edu

Liqiang Chen

University of Nebraska - Lincoln, lche1@unlnotes.unl.edu

Follow this and additional works at: http://aisel.aisnet.org/sighci2005

\section{Recommended Citation}

Nah, Fiona Fui-Hoon; Lee, Hong-Hee; and Chen, Liqiang, "Information Search Patterns in E-Commerce Product Comparison Services" (2005). SIGHCI 2005 Proceedings. 3.

http://aisel.aisnet.org/sighci2005/3 


\section{Information Search Patterns in E-Commerce Product Comparison Services}

\author{
Fiona Fui-Hoon Nah \\ University of Nebraska - Lincoln \\ fnah@unlnotes.unl.edu
}

\author{
Hong-Hee Lee \\ University of Nebraska - Lincoln \\ honghlee@bigred.unl.edu
}

\author{
Liqiang Chen \\ University of Nebraska - Lincoln \\ lche1@unlnotes.unl.edu \\ lche1@unlnotes.unl.edu
}

\begin{abstract}
The presentation of product information is very important in e-commerce websites. In this research, we study how disposition styles can influence users' search patterns in product comparison services of e-commerce websites. Our results show that people are inclined to use feature information paths in vertical disposition style and product information paths in horizontal disposition style. The results also indicate that there are more feature paths than product paths in the earlier stage of product comparison, and more product paths than feature paths in the latter stage of product comparison. Based on Gensch's two-stage choice model and the results of our study, the vertical disposition style is more suited for supporting product comparison services.
\end{abstract}

\section{Keywords}

Product comparison, information paths, e-commerce.

\section{INTRODUCTION}

The market share of e-commerce has increased dramatically in the last decade. Major U.S. retail stores like Wal-Mart and Sears have established a brick-and-click strategy to enhance their competitive advantage. The value of e-commerce is derived from product comparison services where customers can compare a wide variety of alternative products at their convenience and at the comfort of their homes (Keeney, 1999).

The design of e-commerce websites to support product comparison services can influence online traffic and sales (Lohse and Spiller, 1998). It is important for firms to help customers find the products or information they need in order to increase online sales and promote return visits to their websites. One strategy to accomplish this objective is to implement an effective information presentation format for product comparison on e-commerce websites.

Product comparison services in e-commerce websites are usually presented in one of two disposition styles: vertical disposition and horizontal disposition. Vertical disposition displays products by columns and features/attributes by rows (see Figure 1), whereas horizontal disposition displays products by rows and features/attributes by columns (see Figure 2). For example, vertical disposition style is used for product comparison at Dell.com and Canon.com, and the horizontal disposition style is used to display different flight options at Travelocity.com.

\begin{tabular}{|c|c|c|c|c|}
\hline & Product 1 & Product 2 & $\ldots$ & Product N \\
\hline Image & Milk 1 & Milk 2 & $\ldots$ & Milk N \\
\hline $\begin{array}{c}\text { Feature 1 } \\
\text { (Ex. Name) }\end{array}$ & Min \\
\hline $\begin{array}{c}\text { Feature 2 } \\
\text { (Ex. Price) }\end{array}$ & 3.44 & 2.49 & $\ldots$ & 4.25 \\
\hline$\ldots$ & $\ldots$ & $\ldots$ & $\ldots$ & $\ldots$ \\
\hline $\begin{array}{c}\text { Feature N } \\
\text { (Ex. Status) }\end{array}$ & In Stock & In Stock & $\ldots$ & In Stock \\
\hline
\end{tabular}

Figure 1. Vertical Disposition Style in Product Comparison

\begin{tabular}{|c|c|c|c|c|c|}
\hline & Image & $\begin{array}{c}\text { Feature 1 } \\
\text { (Ex. Name) }\end{array}$ & $\begin{array}{c}\text { Feature 2 } \\
\text { (Ex. Price) }\end{array}$ & $\cdots$ & $\begin{array}{c}\text { Feature N } \\
\text { (Ex. Status) }\end{array}$ \\
\hline Product 1 & Milk 1 & 3.44 & $\ldots$ & In Stock \\
\hline Product 2 & $\ldots$ & Milk 2 & 2.49 & $\ldots$ & In Stock \\
\hline $\begin{array}{c}\text { Product } \\
\mathrm{N}\end{array}$ & $\ldots$ & $\ldots$ & $\ldots$ & $\ldots$ & $\ldots$ \\
\hline
\end{tabular}

Figure 2. Horizontal Disposition Style in Product Comparison

Research has shown that information presentation format can influence consumers' information acquisition and processing strategies (Bettman and Kakkar, 1977; Biehal and Chakravarti, 1982; Jarvenpaa, 1989). Hence, the disposition style used in product comparison services can have an impact on consumers' information acquisition patterns and possibly the decisions they make. The objective of this research is to examine the effects of vertical and horizontal disposition styles on users' information search patterns in the e-commerce context.

\section{LITERATURE REVIEW}

Several studies have provided support for the influence of information presentation format on users' browsing and search patterns on websites. For example, using the cognitive fit theory, the 'competition for attention' theory, and the scanpath theory, Hong et al. (2004a) found that specific "presentation formats" support specific online shopping tasks - a list-format display provides better 
support for "browsing" tasks while a matrix-format display facilitates "searching" tasks. This is in line with cognitive fit theory, which suggests that when the problem representation (information representation format) matches the nature of the task, a quicker and more accurate decision making performance can be achieved.

In another study, Hong et al. (2004b) studied the impact of presentation mode and information format on user's interaction with websites. Two presentation modes (textonly versus image-text) and two information formats (list versus array) were compared. Consistent with predictions from the dual coding theory and the proximity compatibility principle, the image-text presentation mode and the list information format were found to outperform the text-only presentation mode and the array information format in terms of information search time, recall of brand names and product images, and attitudes towards the screen design and using the website.

Bettman and Kakkar (1977), Biehal and Chakravarti (1982), and Jarvenpaa (1989) studied the effect of information presentation format on consumers' information acquisition and processing strategies. They found that decision makers' acquisition and processing strategies were influenced by whether information was organized by alternatives or attributes. Information organized by alternatives promotes processing by alternatives whereas information organized by attributes promotes processing by attributes. Both attribute and alternative processing were used when a matrix format was used.

\section{THEORETICAL BACKGROUND \& HYPOTHESES}

Customers' search patterns refer to the patterns of their visual attention. In this research, a customer's visual attention from one target to another is referred to as an information search path. Three types of information search paths are possible in vertical and horizontal disposition styles: feature, product, and crossed. If a customer moves his/her attention from one target to another within the same feature (of different products), the customer follows the feature path. This happens when one is comparing a specific feature/attribute of two products. On the other hand, if a customer moves his/her attention from one target to another across features of the same product, the customer follows the product path. This takes place when one scans two features of a product. When a path is neither a feature or product path, it is a crossed path, which refers to the movement of visual attention to a different feature of a different product. The two information paths that are of interest in this research are feature and product paths.

In the western context, people are accustomed to reading and writing horizontally, from left to right, then from top to bottom. Since perceptual span is smaller in a vertical direction than in a horizontal direction and readers have better control of their eye movements in a horizontal direction (Rayner and Pollatsek, 1989; Ojanpaa et al., 2002), users are more likely to carry out horizontal search patterns than vertical search patterns. Similarly, vertically arranged text is read more slowly than horizontally arranged text (Tinker, 1955; Laarni et al., 2004), thus favoring horizontal patterns of information acquisition. This reading habit, coupled with the larger perception span of horizontal vision, influences people's tendency to browse information horizontally than vertically. Thus, consumers' attention is more likely to shift to another target on the same horizontal row than on the same vertical column. Since feature information across product is organized horizontally in the vertical disposition style but vertically in the horizontal disposition style, there will be more feature information paths in the vertical disposition style than in the horizontal disposition style. Hence, we hypothesize that:

H1: There will be more feature information paths in the vertical disposition style than in the horizontal disposition style.

While product information is displayed horizontally in the horizontal disposition style, the same information is displayed vertically in the vertical disposition style. Since consumers' attention is more likely to shift to another target on the same horizontal row than the same vertical column, the horizontal disposition style will yield more product information paths than the vertical disposition style. Thus, we hypothesize that:

H2: There will be more product information paths in the horizontal disposition style than in the vertical disposition style.

According to Gensch's (1987) two-stage disaggregate attribute choice model, two stages are involved in making a final choice (such as product selection). The first stage is attribute-processing to screen and narrow down the number of alternatives for consideration. The second stage is alternative-processing which considers the attributes simultaneously while allowing for tradeoffs among the attributes. In the first stage, several products are excluded by an initial screening process, and in the second stage, a compensatory analysis is used to derive the final decision (Lehtinen, 1974). In other words, people tend to use a conjunctive strategy to eliminate unacceptable alternatives before they apply a compensatory strategy to evaluate the remaining alternatives and derive a final choice (Lussier and Olshavsky, 1979).

The Gensch's (1987) two-stage choice model can be used to explain product and feature information paths in the first and second stages of the decision-making process on product comparisons. In the first stage, consumers focus on the important features (attributes) by comparing each of these features across the products (i.e., attributeprocessing) to eliminate products (alternatives) that are considered unacceptable or inferior. Hence, feature information paths are used to narrow down the number of products (alternatives) for consideration. In the second stage, customers examine and assess each of the remaining products by making "explicit tradeoffs" among them 
(Payne et al., 1993). Hence, product information paths are used to bind attributes together (alternative-processing) so they can be considered simultaneously in product comparison and in making the final selection.

According to the two-stage choice model, feature information paths (attribute-processing) are mainly used in the first stage. Hence, we hypothesize that more feature information paths will be used in the first stage than in the second stage of the product selection process. Thus,

H3: There are more feature information paths in the first stage than second stage of the product selection process.

Similarly, according to the two-stage choice model, product information paths (alternative-processing) are used mainly in the second stage. Hence, we hypothesize that there are more product information paths in the second stage than in the first stage of the product selection process.

H4: There are more product information paths in the second stage than first stage of the product selection process.

\section{RESEARCH METHODOLOGY}

Figure 3 shows the research model. A within-subject experimental design was used to test the hypotheses. Each subject participated in two sessions in a random order one session with vertical disposition style and the other session with horizontal disposition style - thus serving as his/her own control. The order of disposition styles given to the subjects was randomized to counter-balance any possible ordering effect. Figure 4 shows a screen shot depicting the vertical disposition style.

The matrix format was used for product search and comparison since it facilitates searching tasks (Hong et al., 2004a). To control for the positioning of the products, a randomized procedure was used. For each subject, the total set of 10 products was randomly divided into two sets one set for each disposition style (i.e., 5 products per disposition style). They were then displayed in a completely randomized order in the product comparison matrix. In this way, the placement of products was completely randomized.

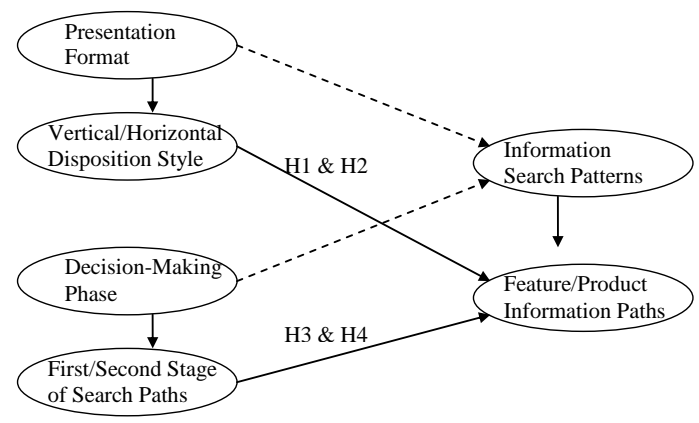

Figure 3. Research Model

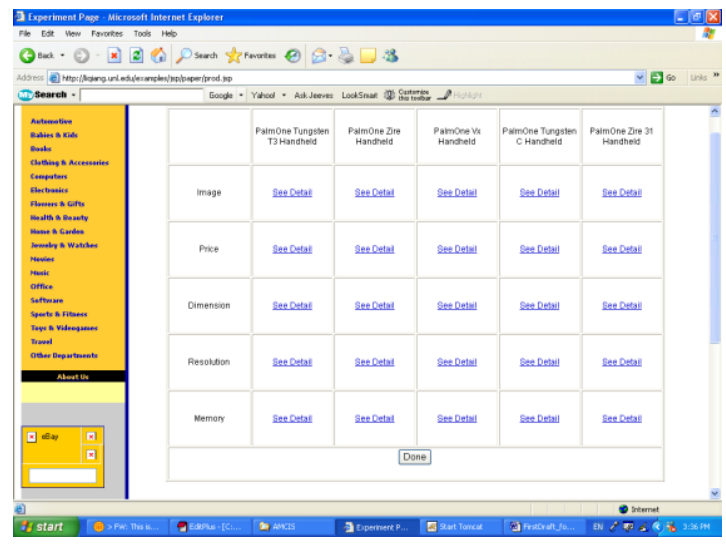

Figure 4. Screen Shot for Vertical Disposition Style

In each session, the subject was asked to select a product that he/she would like to purchase. Each of the cells in the product comparison table contained information about a feature of a product. At the beginning of the experiment, the information in all the cells was hidden. To view the information in a cell, the subject clicked on the cell to have the information displayed. When the subject clicked on another cell, the information on previously opened cells was hidden again (as was done in other studies on consumer choice such as Payne, 1976) in order to precisely track which information was being attended to at any one time and to derive the complete information search paths. This process-tracing method, known as information display boards, has been widely used in the study of consumer choice (Painton and Gentry, 1985; Todd and Benbasat, 1987). Using this method, the visual attention of each subject was tracked using computer logs of the click patterns. The subject was allowed to click on any cell as many times as s/he needed until a product was selected for purchase. Upon completion of the task (i.e., the product to be purchased has been determined), the subject clicked on the "Done" button at the bottom of the screen (see Figure $4)$.

\section{RESULTS}

24 subjects participated in this study. They were students recruited from business and computer science majors. The hypotheses were assessed using repeated-measures ANOVA. Tables 1 and 2 show the descriptive statistics and results for $\mathrm{H} 1$ and $\mathrm{H} 2$. Both $\mathrm{H} 1$ and $\mathrm{H} 2$ are supported at the 0.05 level.

\begin{tabular}{|l|l|l|l|l|}
\hline $\begin{array}{l}\text { Hypothesis } \\
\text { Testing }\end{array}$ & $\begin{array}{l}\text { Disposition } \\
\text { Style }\end{array}$ & $\begin{array}{l}\text { Information } \\
\text { Path }\end{array}$ & $\begin{array}{l}\text { Mean of \# of } \\
\text { Information Paths }\end{array}$ & $\begin{array}{l}\text { Std. } \\
\text { Dev. }\end{array}$ \\
\hline \multirow{2}{*}{ H1 } & Vertical & Feature & 18.23 & 13.52 \\
\cline { 2 - 3 } \cline { 4 - 5 } & Horizontal & & 13.18 & 8.45 \\
\hline \multirow{2}{*}{ H2 } & Vertical & \multirow{2}{*}{ Product } & 5.55 & 5.74 \\
\cline { 2 - 3 } & Horizontal & & 8.14 & 7.53 \\
\hline
\end{tabular}

Table 1. Descriptive Statistics for Testing H1 and H2 


\section{Table 2. Test Results for $\mathrm{H} 1$ and $\mathrm{H} 2$}

Since it is not possible to determine the end of the first stage or the beginning of the second stage (these two stages are also likely to overlap), we have used the halfpoint (in terms of number of clicks) approach to test the number of information paths in the first and second stages. Tables 3 and 4 show the descriptive statistics and results for $\mathrm{H} 3$ and $\mathrm{H} 4$, which are both supported.

\begin{tabular}{|l|l|l|l|l|}
\hline $\begin{array}{l}\text { Hypothesis } \\
\text { Testing }\end{array}$ & Phase & $\begin{array}{l}\text { Information } \\
\text { Path }\end{array}$ & $\begin{array}{l}\text { Mean of \# of } \\
\text { Information Paths }\end{array}$ & $\begin{array}{l}\text { Std. } \\
\text { Dev. }\end{array}$ \\
\hline H3 & First Half & Feature & 18.46 & 11.99 \\
\cline { 2 - 3 } & Second Half & & 15.29 & 7.90 \\
\hline H4 & First Half & Product & 6.67 & 6.04 \\
\cline { 2 - 2 } \cline { 4 - 5 } & Second Half & & 9.50 & 6.87 \\
\hline
\end{tabular}

Table 3. Descriptive Statistics for Testing $\mathrm{H3}$ and $\mathrm{H4}$

\begin{tabular}{|l|l|r|}
\hline \multicolumn{1}{|c|}{ Effect } & F & Sig. (1-tailed) \\
\hline Phases on \# of Feature Information Paths & 6.856 & .01 \\
\hline Phases on \# of Product Information Paths & 2.862 & .01 \\
\hline
\end{tabular}

Table 4. Test Results for $\mathrm{H3}$ and $\mathrm{H4}$

As shown in Table 5, we also examine whether there is any interaction effect between disposition styles (vertical/horizontal) and phases (first half/second half). The results show that there is no moderating or interaction effect.

\begin{tabular}{|c|c|r|r|}
\hline Interaction Effect & $\begin{array}{c}\text { Dependent } \\
\text { Variable }\end{array}$ & F & Sig. (2-tailed) \\
\hline \hline Disposition Styles and Phases & \# of Feature I. P. & 1.054 & .16 \\
\hline Disposition Styles and Phases & \# of Product I. P. & .142 & .36 \\
\hline
\end{tabular}

Table 5. Test Results for Interaction Effect

\section{DISCUSSION OF RESULTS}

All four hypotheses are supported. $\mathrm{H} 1$ and $\mathrm{H} 2$ suggest that people have a tendency to search for information horizontally than vertically, which is in line with their reading habits. Given the horizontal search bias, feature information paths are used more often in vertical than horizontal disposition style whereas product information paths are used more often in horizontal than vertical horizontal style. On the other hand, H3 and H4 suggest that attribute processing or feature information paths are used more frequently in the first half than in the second half of the product selection process. They also suggest that alternative processing or product information paths are employed more often in the second half than first half of the product selection process. This finding is in line with Gensch's two-stage disaggregate attribute choice model, where the first stage involves mainly the use of attribute processing to screen or eliminate unwanted products (alternatives) to a more manageable consideration set while the second stage involves the use of alternative processing to derive at a final choice.
Since information presentation format can bias consumers' patterns of information acquisition, appropriate information presentation format that is congruent with the desired or an effective method of processing can be presented to consumers to promote the use of that processing method. For example, if processing by attribute is desired or is thought to be effective, then vertical disposition style can be used. Since the first stage of decision making on product comparison relies on feature information paths, it seems desirable to use vertical disposition in product comparison services. In fact, many product comparison services in e-commerce websites use vertical disposition style to promote attribute processing across products.

\section{CONCLUSION, LIMITATIONS, \& FUTURE RESEARCH}

The effects of disposition styles on customers' search patterns in product comparison services were examined. The study results show that people are inclined to use feature information paths when they are given the vertical disposition style and product information paths when they are given the horizontal disposition style. In the vertical disposition style, users have a tendency to focus on feature or attribute information when making comparisons among products. Such attribute-based comparisons can be difficult in physical stores. In physical stores, information is sorted by products or brands, which encourages alternative (product/brand) processing. Attribute processing is made feasible by online product comparison services.

According to Payne's decision making theory (Payne et al., 1993) and Gensch's (1987) two-stage choice process, consumers will first compare the feature/attribute information (such as price) of a list of available products to eliminate the undesirable products and to arrive at a smaller set of products for consideration. Next, consumers make their final decision by binding the features of each product together for making comparisons among the products. The experimental results support this two-stage process, where people follow more feature information paths and less product information paths in the first stage than in the second stage. These findings have practical values. In the first stage, consumers need feature information and attribute processing which can be well supported by vertical disposition style. In the second stage, consumers need product information to make a final choice. Based on the study results and the literature on number of products being considered in each stage, vertical disposition is recommended for product comparison services on e-commerce websites.

There are some limitations in this research which call for future research. First, in this study, we tracked and recorded subjects' movement of visual attention using the information display board method that is widely used in consumer choice research. This method is appropriate for this study because it allowed us to capture the movement of visual attention precisely in order to accurately determine the type of information paths used by the 
subjects. However, we acknowledge that not all visual attention might be translated into information processing. This is a limitation of the information display board method. In future research, we plan to conduct the same study using the 'think-aloud' protocol (i.e. by asking subjects to think aloud during the process) to gain a better understanding of consumers' decision making processes. The think-aloud protocol method was not used in this study because the current study focuses on information acquisition and search patterns rather than the cognitive process of decision making. Second, we have limited the number of attributes and number of products to 5 in this study because they fit within the $7 \pm 2$ rule representing the short-term capacity of human information processing (Miller, 1956). In future research, we are interested in extending this study to examine product comparison services for a larger set of products and attributes. Third, the generalizability of the study may be limited. The information search paths of subjects may be influenced by their expertise and product familiarity (Bettman and Kakkar, 1977).

In summary, this study provides a theoretical view of how disposition styles impact customers' information search patterns. It also demonstrates how disposition styles can be used to influence the information acquisition strategies of decision makers. That is, if there is a desired method of processing information (i.e., an easier or more effective method), the information can be presented in a disposition style that is congruent with that method of processing. This research also provides some useful guidelines on the design of product comparison services on e-commerce websites.

\section{REFERENCES}

1. Bettman, J.R. and Kakkar, P. (1977) Effects of Information Presentation Format on Consumer Information Acquisition Strategies, Journal of Consumer Research, 3, 233-240.

2. Biehal, B. and Chakravarti, D. (1982) InformationPresentation Format and Learning Goals as Determinants of Consumers' Memory Retrieval and Choice Processes, Journal of Consumer Research, 8, 431-441.

3. Gensch, D. H., (1987) A Two-Stage Disaggregate Attribute Choice Model, Marketing Science, 6, 3, 223239.

4. Hong, W., Thong, J. Y. L., and Tam, K. Y. (2004a) The Effects of Information Format and Shopping Task on Consumers' Online Shopping Behavior: A Cognitive Fit Perspective, Journal of Management Information Systems, 21, 3, 149-184.
5. Hong, W., Thong, J. Y. L., and Tam, K. Y. (2004b) Designing Product Listing Pages on E-commerce Websites: An Examination of Presentation Mode and Information Format, International Journal of HumanComputer Studies, 61, 4, 481-503.

6. Jarvenpaa, S.L. (1989) The Effect of Task Demands and Graphical Format on Information Processing Strategies, Management Science, 35, 3, 285-303.

7. Keeney, R.L. (1999) The Value of Internet Commerce to the Customer, Management Science, 15, 4, 533542.

8. Laarni, J., Simola, J., Kojo, I., and Risto, N. (2004) Reading Vertical Text from a Computer Screen, Behaviour and Information Technology, 23, 2, 75-82.

9. Lehtinen, O. (1974) A Brand Choice Model: Theoretical Framework and Empirical Results, Journal of European Research, 2, 51-68.

10. Lohse, G.L. and Spiller, P. (1998) Electronic Shopping, Communications of the ACM, 41, 7, 81-87.

11. Lussier, D.A. and Richard W.O. (1979) Task Complexity and Contingent Processing in Brand Choice, Journal of Consumer Research, 6, 154-165.

12. Miller, G. (1956) The Magical Number Seven, Plus or Minus Two, Psychological Review, 63, 81-97.

13. Ojanpaa, H., Nasanen, R, and Kojo, I. (2002) Eye Movements in the Visual Search of Word Lists, Vision Research, 42, 1499-1512.

14. Painton, S. and Gentry, J.W. (1985) Another Look at the Impact of Information Presentation, Journal of Consumer Research, 12, 2, 240-244.

15. Payne, J.W. (1976) Task Complexity and Contingent Processing in Decision Making: An Information Search and Protocol Analysis, Organizational Behavior and Human Performance, 16, 2, 366-387.

16. Payne, J. Bettman, J. and Johnson, E.(1993) The Adaptive Decision Maker, Cambridge Univ. Press.

17. Rayner, K. and Pollatsek, A. (1989) The Psychology of Reading, Prentice-Hall.

18. Tinker, M. A. (1955) Perceptual and Oculomotor Efficiency in Reading Materials in Vertical and Horizontal Arrangements, American Journal of Psychology, 68, 444-449.

19. Todd, P. and Benbasat, I. (1987) Process Tracing Methods in Decision Support Systems Research: Exploring the Black Box, MIS Quarterly, 11, 4, 493512. 